\title{
RIESGOS Y SEGUROS DE RC DURANTE LA CONSTRUCCIÓN Y OPERACIÓN DE GRANDES PROYECTOS
}

\section{RISKS AND LIABILITY INSURANCES DURING THE CONSTRUCTION AND OPERATION OF LARGE- SCALE PROJECTS}

\author{
NANCY ARIAS MORA** \\ Fecha de recepción: 16 de octubre 2019 \\ Fecha de aceptación 30 noviembre 2019 \\ Disponible en línea: 30 de diciembre 2019
}

Para citar este artículo/To cite this article

Arias Mora, Nancy, Riesgos y Seguros de RC durante la construcción y operación de grandes proyectos, 51 Rev.Ibero-Latinoam.Seguros, 111-126 (2019). https://doi.org/10.11144/Javeriana.ris51.rsrc

doi:10.11144/Javeriana.ris51.rsrc

Magister en Derecho Público. Consejo Universitario de la Universidad Estatal a Distancia, Asesora Legal 


\section{RESUMEN}

El ensayo se presenta como el resultado de un análisis crítico de algunos casos representativos del manejo de la Responsabilidad Civil en los Tribunales de Justicia de Costa Rica. Para ello se ha incorporado el análisis de casos tramitados en otros países, en los cuales se resuelven situaciones similares, pero con resultados distintos. La propuesta es que las acciones de otros países sirvan como referencia, como lecciones aprendidas. El aporte de este análisis es referencial e intenta hacer un incentivo para el aprendizaje y asesoría en materia de seguros y administración de riesgos, para lograr una buena gestión de los mismos, en particular y especialmente, lo que se refiere a los posibles daños a terceros causados en la construcción o por Responsabilidad Civil.

Palabras clave: responsabilidad civil, seguro de Responsabilidad Civil, Costa Rica, patrimonio cultural, grandes proyectos. 


\begin{abstract}
The essay is presented as the result of a critical analysis of some representative cases of Liability management in Costa Rica's Courts of Justice. This also includes the analysis of cases from other countries in which similar situations are resolved with different results. The proposal is that those actions from other countries are used as references and lessons learned. The contribution of this analysis is referential and attempts to act as an incentive for learning and assessment in the field of insurance and risk management to achieve their proper administration, particularly and especially for possible third-party damages caused by or resulting from assessing Liability.
\end{abstract}

Keywords: liability, Liability insurance, Costa Rica, cultural heritage, largescale projects.

\title{
SUMARIO:
}

INTRODUCCIÓN. 1. LOS CONTRATOS DE CONCESIÓN EN EL ESTADO. 2. SOBRE LOS SEGUROS DE RESPONSABILIDAD CIVIL. 3. CASOS PRÁCTICOS DE RESPONSABILIDAD CIVIL EN GRANDES PROYECTOS DE EJECUCIÓN PÚBLICA Y PRIVADA (COSTA RICA Y OTROS PAÍSES). CONCLUSIONES. BIBLIOGRAFÍA. 


\section{INTRODUCCIÓN}

El análisis de casos sobre grandes proyectos de ejecución de obras tanto públicas como privadas, es el tema central de esta ponencia que responde a la correlatoría por Costa Rica en el marco del Congreso Ibero Latinoamericano de Derecho en Seguros Lima, 2019 (Díaz-Granados, JM (2019, agosto) Riesgos y Seguros de RC durante la construcción y operación de grandes proyectos, presentado en XVI Congreso Ibero Latinoamericano de Derecho en Seguros, Lima, Perú)

Corresponde hacer de previo algunos apuntes sobre la ponencia anterior, que me permiten poner una introducción al análisis de casos reales en los cuales podemos ver de forma aplicada, y en situaciones concretas, las diferentes formas de enfrentar un siniestro con un buen seguro o sin él. Posteriormente se hará referencias a casos concretos, sus similitudes y diferencias y algunas observaciones a las situaciones que rodearon los mismos para tomar conciencia y hacer de los ejemplos una base de construcción de experiencia para el Seguro de Responsabilidad Civil en grandes construcciones. Finalmente presentaré conclusiones para dar un enfoque final a la ponencia con reflexiones generales.

\section{LOS CONTRATOS DE CONCESIÓN EN EL ESTADO}

En la ponencia anterior se hizo referencia a los casos de grandes construcciones que derivan de los contratos de concesión de los Estados, ya que éstas son las principales fuentes jurídicas de contratos para infraestructura en nuestros países Latinoamericanos.

Ciertamente, las concesiones han sido utilizadas en los últimos años como la forma clásica de construcción, porque resulta mucho más favorable en cuanto a tiempos de cumplimiento y a facilidades de contratación, que la forma de construcción utilizando los propios medios del Estado.

Específicamente se mencionan en el mapa de responsabilidades y afectaciones, los diferentes tipos de responsabilidad a saber: contractual y extracontractual así como la modalidad de responsabilidad subjetiva y objetiva haciendo referencia a la existencia de culpa o no en la acción dañosa.

Sobre estas afirmaciones y referencias contenidas en el mapa presentado en la ponencia anterior quisiera mencionar, que si bien es cierto la responsabilidad se puede imputar como derivada de un contrato 
(contractual) o no (extra contractual), lo cierto es que, cuando se trata de la construcción u operación de una obra de infraestructura las posibilidades de generar una responsabilidad, en cualquiera de las dos vías, es muy grande. Pero como se trata de la operación de obras a cargo del Estado, la responsabilidad siempre será imputable a ésta. Con las salvedades de ley mencionadas, que son las únicas que tienen la virtud de romper el nexo causal de responsabilidad, que en Costa Rica son la fuerza mayor, la culpa de la víctima, el hecho de un tercero y está así dispuesto en los artículos 190 y siguientes de la Ley General de Administración Pública (Ley General de Administración Pública, No 6227).

Es decir, cuando se trata de obras a cargo del Estado, éste siempre será responsable de los daños que se causen aún y cuando la obra haya estado otorgada en concesión a un privado para su ejecución, ya que, en Costa Rica, existiendo la Ley de Concesiones de Obra Pública, se deja claro que el Estado concesiona la obra, pero no cede ni renuncia a su responsabilidad.

Siendo así, salvo que se rompa el nexo causal con las eximentes de responsabilidad citadas, el Estado siempre será responsable por los daños causados en dichas obras. Claro que podrá, en todos los casos, derivar responsabilidades a quienes ha concesionado las obras en tanto su participación tenga relación con los daños, y podrá ejecutar las garantías que evidentemente habrá obtenido en virtud de la concesión, pero su responsabilidad primaria siempre existirá.

\section{SOBRE LOS SEGUROS DE RESPONSABILIDAD CIVIL}

Se mencionó en la ponencia anterior la función dual de este seguro, una de ellas en protección del patrimonio del asegurado y la otra para cubrir el daño causado a un tercero.

Ciertamente estas son las dos funciones básicas del Seguro de Responsabilidad Civil, que para estas obras se vuelve indispensable contar con él y que además responda a una buena asesoría sobre los posibles riesgos y con un buen monto asegurado, con coberturas apropiadas según la actividad y la construcción, haciendo siempre la observación de que el monto, en este tipo de seguros, nunca será exacto y en muchas ocasiones es insuficiente dada la imprevisibilidad de los daños que puedan derivarse de la obra.

Un seguro que haya sido producto de una buena inspección y que tenga asesoría en cuanto a los posibles daños que deban cubrirse, va a res- 
ponder claramente a la función dual que tiene, ya que podrá responder a una posible afectación al patrimonio del asegurado al indemnizar el daño, al menos en un porcentaje suficiente para que el asegurado no vea afectado su patrimonio ni la operación de su negocio y al mismo tiempo tendrá la posibilidad de responder al eventual daño que cause a un tercero e indemnizarle para saldar su responsabilidad. Reitero aquí la necesidad de que se adquiera el seguro con asesoría correcta y profesional que le garantice al asegurado, que según lo dicho antes, podría ser el Estado o más bien lo es en muchos de los casos, el resarcimiento del daño y la satisfacción de las obligaciones derivadas de cualquier evento que genere responsabilidad.

En Costa Rica, la operación del Seguro de Responsabilidad Civil se encuentra descrita de manera general en la Ley Reguladora del Contrato de Seguros (Ley No 8956, Diario Oficial La Gaceta No 175 de la República de Costa Rica, 2011, artículo 88) y no permite la acción directa contra el asegurador. Esta discusión doctrinaria permite que en algunos países la regulación tenga dispuesta la acción directa, sin embargo, en Costa Rica, a pesar de que fue un tema que se ha discutido en varias oportunidades, no se ha variado el sistema y la normativa vigente no solo no permite la acción directa ante el asegurador, sino que la prohíbe.

Otro tema del que se habló en la ponencia anterior es la necesidad de que el asegurado sea responsable para que la cobertura del seguro pueda ser aplicada, sin embargo, vale la pena hacer la aclaración de que en algunas legislaciones, la aplicación del seguro permite la posibilidad de llegar a arreglos previos con el tercero perjudicado. Se habla de arreglos previos porque se trata de llegar a una conciliación de previo al dictado de una sentencia condenatoria en sede judicial, y con ello dar por terminado el proceso judicial y cualquier otra reclamación sobre el evento.

En esos casos, en los que sea posible llegar a un arreglo anticipado, no es necesario contar con una declaratoria de responsabilidad directa de parte del asegurado ya que la legislación penal en Costa Rica permite conciliar antes del dictado de la sentencia obtener una extinción de la acción penal. Así las cosas, existe como excepción a la regla general de que deba el asegurado tener una declaratoria de culpa o de responsabilidad expresa. Claro está que eso no elimina la obligación de que sí exista responsabilidad en el evento y que además debe ser evidente, ya que es un análisis previo que debe hacer la aseguradora.

En este aparte quisiera mencionar también que, ciertamente para lograr los Objetivos de Desarrollo Sostenible (ODS), los países tendrán que aprovechar al máximo sus recursos para el desarrollo, usando no solo 
fondos públicos sino también financiamiento privado y soluciones del sector privado sostenibles. Y esta es una de las principales razones por las cuales se acude a la figura de la concesión en nuestro países, pero en ese caso, debemos definir con mucho mayor espero, cuidado y asesoría profesional la responsabilidad de cada parte en los proyectos y la suscripción de los seguros que garanticen su protección para el patrimonio del Estado y para los eventuales terceros afectados.

\section{CASOS PRÁCTICOS DE RESPONSABILIDAD CIVIL EN GRANDES PROYECTOS DE EJECUCIÓN PÚBLICA Y PRIVADA (COSTA RICA Y OTROS PAÍSES)}

\footnotetext{
- Autopistas del Sol, Chile (The cliniccl (2013) recuperado el 27 de junio de 2019 de https://www.theclinic.cl/2013/02/07/suprema-condena-a-autopista-del-sol-a-pagar-115-millones-de-indemnizacion-por-accidente-con-caballos/).
}

El primer caso que se expone corresponde a un evento de Autopistas del Sol en Chile en el que la Corte Suprema condena a Autopista del Sol a pagar $\$ 115$ millones de indemnización por accidente con caballos. En este caso, el que se cita a efectos de comparar la resolución judicial de Chile con la eventual resolución que se daría en Costa Rica, la Corte Suprema condenó a la concesionaria Autopista del Sol S.A. a pagar la suma de 115.000.000 de pesos a los integrantes de un grupo familiar que resultaron con serias lesiones al embestir con su vehículo a unos caballos que se atravesaron en la vía. En un fallo unánime, los ministros de la Sala Constitucional del máximo tribunal del país determinaron que la concesionaria debía cancelar la millonaria suma a Julio Gómez Zúñiga, su esposa e hija por un accidente sufrido el 11 de octubre de 2004 a la altura del kilómetro 80 de la vía. La sentencia rechazó la demanda en contra del Estado de Chile en el accidente al considerar que no existe responsabilidad por una eventual falta de fiscalización, ya que los contratos de concesión otorgan a las empresas la obligación de mantener condiciones de seguridad para evitar estos hechos.

A contrario de lo que podría ocurrir en Costa Rica, en la sentencia de la Suprema Corte de Chile, se exonera al Estado porque la concesionaria asume todo el riesgo, sin considerar que la concesión no es una eximente de responsabilidad objetiva, la cual corresponde a la teoría de la exposición al riesgo, es decir, siendo el Estado quien da el proyecto en concesión, no pierde nunca la responsabilidad sobre el mismo de acuerdo a las funciones que le corresponden. 
Dos casos en Costa Rica nos ayudarán a comprender mejor esto.

- Caso del Puente de Turrubares, Costa Rica (Periódico La Nación recuperado el 25 de julio de 2019 de www.nacion.com/sucesos/ cinco-personas-fallecen-al-caer-puente-entre-turrubaresyorotinal T6E5PDJJSZEZ5OFRBWFBYQ3OTY/story/).

Es un sitio cerca de San José en el cual, en un pueblo se unían dos poblados por medio de un puente de hamaca, es decir, un puente de cables que había sido puesto temporalmente mientras el Estado construía un puente formal. Se trata de una vía por la cual transitaban vehículos normalmente con el único cuidado de que solo podía circular un vehículo a la vez. Todos conocían el riesgo de utilizarlo, pero era la única vía para transitar. A pesar de que el Estado es el responsable de mantener las vías públicas en buen estado si se trata de vías nacionales y las $\mathrm{Mu}-$ nicipalidades (Gobiernos Locales) las vías cantonales, este puente pasó muchos años con esa situación hasta que ocurrió la tragedia.

En Costa Rica la responsabilidad del Estado está claramente definida en la Ley General de Administración Pública (Ley General de Administración Pública, Ley No 6227, Costa Rica. Artículo 190. “1. La Administración responderá por todos los daños que cause su funcionamiento legítimo o ilegítimo, normal o anormal, salvo fuerza mayor, culpa de la víctima o hecho de un tercero. 2. La Administración será responsable de conformidad con este artículo, aun cuando no pueda serlo en virtud de las secciones siguientes de este Capítulo, pero la responsabilidad por acto lícito o funcionamiento normal, se dará únicamente según los términos de la Sección Tercera siguiente"), razón suficiente para considerar la inmensa obligación del Estado de proteger su patrimonio con un Seguro de Responsabilidad Civil.

Este caso se expone porque es una gran obra de infraestructura que no se construyó definitivamente, sino que se le dio una solución temporal, con un riesgo muy alto y que además perduró en el tiempo sin mantenimiento ni construcción. En octubre del año 2009 circulaba un autobús lleno de pasajeros, la estructura no soportó el paso del autobús y colapsó, causando la caída del automotor al Río Tárcoles y la muerte de cinco personas, mientras que otras 30 resultaron heridas.

El Estado usualmente no posee Seguro de Responsabilidad Civil y en este caso no hubo un respaldo para cubrir las lesiones y muerte causadas por el accidente. Se acusó penalmente a la Ingeniera de Puentes del Ministerio de Obras Públicas y Transportes (Mopt) y al chofer del bus. 
Precisamente el próximo 22 de octubre del 2019 se cumplirán 10 años de esta tragedia sin que a este momento se haya logrado indemnizar a las víctimas del fatal accidente. El Estado no tenía con qué asumir los daños y todas las previsiones, el traslado y administración de los riesgos, en este caso fallaron. No se contó con ningún Seguro ni con la diligente acción del Estado, responsable, para indemnizar a los terceros, del daño percibido.

- Caso del Redondel de Toros, Turrialba, Costa Rica (Periódico La Nación recuperado el 26 de junio de 2019 de https://www.nacion.com/el-pais/varias-fallas-en-el-redondel-eturrialba/UQUOC26LF5GFLC2JQROTSYU4KE/story/)

Este caso es ejemplarizante y se cita porque a más de 10 años de ocurrido el evento y 14 años después de haberse dictado sentencia en este caso, se mantiene la teoría de responsabilidad civil indirecta, por omisión, por culpa in eligendo e in vigilando y una condena solidaria que nos comprueba que en el tema de Responsabilidad Civil es impredecible lo que se pueda condenar en sentencia.

El evento ocurre en 1998 y se resuelve en sede judicial, de forma definitiva en el año 2005. Se desploma un Redondel de Toros, que es una gran construcción que puede albergar unas 200 personas para disfrutar de las corridas de toros, que es una actividad tradicional de los pueblos en Costa Rica. Son grandes construcciones que están supervisadas y controladas por varias instancias el Estado.

Este caso es icónico porque el Redondel colapsa y cientos de personas caen, llevando la peor parte un joven menor de edad que se encontraba debajo de la estructura, viendo las corridas de toros desde un sitio no habilitado, y al momento del colapso, gran parte de la estructura cae sobre todo su cuerpo causándole una gravísima lesión en su columna vertebral dejándolo cuadripléjico.

Lo relevante de este caso es que sí se contaba con un Seguro de Responsabilidad Civil -porque es obligatorio por tratarse de un espectáculo público- pero con un monto de 1 millón de colones. La acción judicial se lleva adelante por los padres del joven lesionado y entre los demandados se incluyó el Estado en varios de sus participantes (el Ministerio de Salud, el Ministerio de Seguridad Pública, la Municipalidad de Turrialba y el INS como aseguradora estatal) y otros privados. La sentencia en firme condena a todos por partes iguales a responder por los daños causados al menor y a sus padres con la teoría de que se trató una responsabilidad civil indirecta. 
A pesar de que se tenía un Seguro de Responsabilidad Civil, el Despacho Judicial consideró que la aseguradora es también responsable por no haber asegurado por un monto más ajustado a la realidad de los posibles daños y condena a todos por 10 veces el monto asegurado. Se aseguró el evento en aproximadamente $\$ 1800$ (mil ochocientos dólares estadounidenses) y la condena fue de aproximadamente $\$ 18.000$ (dieciocho mil dólares estadounidenses). De la resolución emitida por la Sala Primera de la Corte Suprema de Justicia se resalta el siguiente texto:

“...en la responsabilidad indirecta, se contemplan las hipótesis, de una responsabilidad cometida por una falta de vigilancia y de cuidado con respecto a los sujetos a los que tiene a su cargo un sujeto determinado. Ej. El caso del padre con respecto a sus hijos menores, o el director de una escuela en relación con los alumnos de su escuela. Aquí, el sujeto que ocasiona el daño, no es el mismo que responderá por los daños y perjuicios ocasionados a la víctima." (...)

"En el caso bajo estudio, de los autos se desprende sin lugar a dudas que la Asociación Hogar de Ancianos San Buenaventura incurrió en una culpa in eligendo al no tomar las medidas concernientes en la regulación del actuar del señor Tortós Trejos contratado por ella para la realización de la construcción del redondel; y por otra parte, este último incurrió en una culpa in vigilando al no constatar que dicho redondel se construyera adecuadamente y con los materiales requeridos para que pudiesen llevarse a cabo las corridas de toros adecuadamente y soportara esa estructura el peso de los espectadores que llegarían a observar esos espectáculos" (Exp: 00-000717-163-CA Resolución: 000308-F-2006 SALA PRIMERA DE LA CORTE SUPREMA DE JUSTICIA. San José, a las diez horas treinta minutos del veinticinco de mayo del dos mil seis confirma la sentencia No. 10-2005, a las 13 horas 50 minutos del 4 de marzo del 2005 del Tribunal Contencioso Administrativo, Sección Cuarta)

Este caso es ejemplarizante y comprueba que la relevancia de contar con un buen Seguro que permita restablecer los daños a terceros y que proteja el patrimonio de quienes, en virtud de su participación en eventos, actividades, construcciones o cualquier forma de grandes dimensiones que pueda causar daños a terceros. 
- Otros casos. Japón (World Bank recuperado el 26 de julio de 2019 de https://blogs.worldbank.org/es/voices/app-para-aumentar-la-resiliencia-de-la-infraestructura-que-nos-ensena-japon)

En muchas ocasiones, mantener una buena gestión de riesgos y satisfacer sus obligaciones, no es una situación fácil para el Estado, por lo que debe recurrir a alianzas con otras instituciones o con entidades privadas. A pesar de que esta no es una situación fácilmente aceptada en nuestros país, especialmente por los grandes casos de corrupción en Latinoamérica, en ocasiones es la única opción viable para dar cumplimiento a las obligaciones. Japón es un caso de éxito que cito solo como referencia.

Un consorcio privado firmó una concesión de 30 años para administrar el aeropuerto de Sendai, convirtiéndose en el primer aeropuerto estatal de Japón manejado por el sector privado.

A pesar de ser una zona con gran impacto de desastres naturales, algunas empresas privadas lograron entrar con su negocia a coadyuvar al Estado, siendo un gran ejemplo de co creación de soluciones que respondan a las necesidades de los ciudadanos y satisfagan las obligaciones del Estado pero que a la vez distribuyan, administren y cedan los riesgos, para que las actividades sean exitosas para todos los participantes.

\section{- Otros temas relevantes.}

Otros temas que deben considerase al momento de analizar este tipo de Seguros de Responsabilidad Civil para grandes proyectos son los siguientes:

- Las tarifas. El cálculo de las tarifas de estos seguros, es un gran reto para las asegurados ya que, según lo he expuesto en otras oportunidades, la diferencia de este Seguro en relación con otros Seguros, es la imposibilidad de conocer una aproximación del costo de los posibles daños y solo se podrá hacer mediante la utilización de modelos estadísticos que intenten hacer un ejercicio aproximado con los datos históricos que se tengan. A pesar de tener un monto asegurado, los daños pueden exceder por mucho ese monto o el número de terceros lesionados o dañados puede ser de tal magnitud que no sea posible contar con suficiente cobertura. Es un tema de gran importancia tanto para las aseguradoras como para los asegurados, ya que tendrá un impacto, positivo o negativo para uno u otro, ante un posible evento.

- Edificios Históricos, Patrimonio Cultural, Histórico o de la Humanidad. El aseguramiento de estos edificios, que son grandes 
proyectos y a los que ingresan miles de visitas anualmente, generan un gran riesgo que usualmente no está cubierto. Son proyectos de gran valor y normalmente se mantienen con cargo al Estado, lo que hace más probable que no se cuente con un seguro que les garantice la restitución de cualquier daño que se cause a terceros. Es un tema al que debemos ponerle atención y constituye un mercado a cubrir para la industria aseguradora.

- Notre Dame. El reciente incendio ocurrido en la emblemática Notre Dame ha generado un ejemplo de la necesidad de cubrir estos proyectos. A pesar de que en este caso el responsable del daño podría ser el tercero contratado para realizar las reparaciones, lo cierto es que, pudo ser una tragedia con graves consecuencias, sin considerar el daño que se genera a la cultura, la historia y el turismo a nivel mundial.

- Edificio del Ministerio de Cultura. Este es otro ejemplo en Costa Rica, es el edificio en el que antes se albergaba la Fábrica Nacional de Licores (Fanal). Es un edificio protegido por ser Patrimonio Nacional, en el cual hace muchos años se dio un incendio de grandes dimensiones. Cuando en él se albergaba la Fanal y se manipulaban los componentes químicos - combustibles- propios de la fabricación de licor causaron el incendio, pero pudieron ocasionar un evento de mayores dimensiones ya que se encuentra rodeado de una escuela, viviendas y otros edificios. Estos edificios son responsabilidad del Estado y lo usual es que no cuenten con Seguros de Responsabilidad Civil. Como lo he hecho en otras oportunidades, insto a los intermediarios y aseguradoras a destinar esfuerzos a este segmento de mercado, tan relevante, que se encuentran sin asesoría profesional en relación con este importante seguro.

- El Teatro Nacional de Costa Rica. Este es un edifico icónico, maravilloso que engalana nuestra capital, se le considera uno de los inmuebles más importantes de la historia nacional, y principal joya arquitectónica de la ciudad de San José. Ha sido declarado Monumento Nacional Patrimonio Arquitectónico de Costa Rica. En los últimos años ha generado una gran discusión en Costa Rica por el mantenimiento que necesita para evitar un colapso o generar una emergencia. Es visitado diariamente por cientos de personas ya que cuenta con 
un recorrido por sus salas y obras de arte que le componen, lo cual genera una gran exposición al riesgo de causar un daño (teoría de la exposición al riesgo). Es un ejemplo más de la necesidad de dar cobertura a estas grandes edificaciones con una exposición de riesgo tan alto y que son responsabilidad de los Estados.

- Daños de Impacto Ambiental: En Costa Rica, como en otros países, contamos con el derecho constitucionalmente tutelado a un medio ambiente sano y ecológicamente equilibrado (" $E l$ Estado procurará el mayor bienestar a todos los habitantes del país, organizando y estimulando la producción y el más adecuado reparto de la riqueza. Toda persona tiene derecho a un ambiente sano y ecológicamente equilibrado. Por ello, está legitimada para denunciar los actos que infrinjan ese derecho y para reclamar la reparación del daño causado. El Estado garantizará, defenderá y preservará ese derecho. La ley determinará las responsabilidades y las sanciones correspondientes" Artículo 50 Constitución Política de Costa Rica). Esto debería ser suficiente motivo para que el Estado estuviera protegido por un excelente Seguro de Responsabilidad Civil. Pero también debe ser una razón para que los particulares, que ejercen actividades comerciales se protejan por los eventuales daños que causen en el medio ambiente. Este tema ha estado en discusión por muchos años y aún no se ofrecen coberturas suficientes para estos daños (dejaré pendiente la discusión de si los Seguros Paramétricos están siendo la solución para estos casos) y los eventos siguen ocurriendo.

- Químicos Holanda, Incendio en Moín, Limón, Costa Rica, 2006 (Periódico La Nación recuperado el 27 de julio de 2019 de https://www.nacion.com/el-pais/servicios/estado-pide-c-3-500-millones-a-quimicos-holanda-por-danos/ Y3FIFBEFHFDANLHYXQT4X3PKHI/story/) El Estado Costarricense reclamó $₫ 3.500$ millones a la empresa por daños ambientales causados con el incendio. Este es un caso hubo dos fallecidos y decenas de personas con problemas respiratorios. Adicionalmente, también producto del incendio, murió gran cantidad de flora y fauna y aproximadamente 20.000 habitantes sufrieron dos meses de suspensión del agua potable. Es por estos casos, que menciono el tema como relevante y merecedor de atención por parte de la industria aseguradora. 


\section{CONCLUSIONES}

La principal conclusión a la que debo arribar es que como participantes de la industria de seguros, en cada uno de nuestros países y en la esfera de influencia en que nos desempeñemos, debemos educar en responsabilidad civil, tenemos la obligación de inculcar la costumbre de adquirir seguros. Que esta educación se cumpla como una responsabilidad y no solo porque en el ámbito de las autorizaciones estatales es forzoso contar con un Seguro que responda por los daños que se generen en las grandes construcciones.

No podemos olvidar que es posible que el Estado siempre sea responsable por derivación legal, por lo que debemos promover seguros para el Estado, la industria debe generar propuestas para el Estado, se deben recomendar seguros con un muy buen análisis del riesgo que le permitan a los Estados tener una cobertura, al menos medianamente suficiente para enfrentar cualquier evento de grandes dimensiones. Tenemos que prevenir, que educar en prevención, y siempre contar con buena asesoría y excelente cobertura tanto en grandes construcciones en proceso como en grandes edificaciones en operación.

Los riesgos son controlables, se administran y se trasladan pero en Responsabilidad Civil es impredecible qué ocurrirá y qué daños se generarán, por eso es muy importante contar con las experiencias de otros países para que sirvan de lecciones aprendidas para la industria y generen mayores y mejores contratos de protección para los riesgos de Responsabilidad Civil.

\section{BIBLIOGRAFÍA}

Constitución Política de Costa Rica, 1949

DÍAZ-GRANADOS, JM. (2019, agosto). Conferencia: Riesgos y Seguros de RC durante la construcción y operación de grandes proyectos, presentado en XVI Congreso Ibero Latinoamericano de Derecho en Seguros, Lima, Perú)

Ley Reguladora del Contrato de Seguros, Ley No 8956, Costa Rica

Ley General de Administración Pública, No 6227, Costa Rica

Periódico La Nación recuperado el 27 de julio de 2019 de https://www.nacion.com/elpais/servicios/estado-pide-c-3-500-millones-a-quimicos-holanda-por-danos/Y3FIFBEFHFDANLHYXQT4X3PKHI/story/ 
Periódico La Nación recuperado el 25 de julio de 2019 de www.nacion.com/sucesos/ cinco-personas-fallecen-al-caer-puente-entre-turrubaresyorotina/T6E5PDJJSZEZ5OFRBWFBYQ3OTY/story/)

Periódico La Nación recuperado el 26 de junio de 2019 de https://www.nacion.com/ el-pais/varias-fallas-en-el-redondel-deturrialba/UQUOC26LF5GFLC2JQROTSYU4KE/story/)

Resolución Judicial Expediente $N^{\circ}$ 00-000717-163-CA Voto Nº00308-F-2006 Sala Primera de la Corte Suprema de Justicia. San José, a las diez horas treinta minutos del veinticinco de mayo del dos mil seis confirma la sentencia No. 10-2005, a las 13 horas 50 minutos del 4 de marzo del 2005 del Tribunal Contencioso Administrativo, Sección Cuarta.

The cliniccl (2013) recuperado el 27 de junio de 2019 de https://www.theclinic. $\mathrm{cl} / 2013 / 02 / 07 /$ suprema-condena-a-autopista-del-sol-a-pagar-115-millones-de-indemnizacion-por-accidente-con-caballos/

World Bank recuperado el 26 de julio de 2019 de https://blogs.worldbank. org/es/voices/app-para-aumentar-la-resiliencia-de-la-infraestructura-que-nos-ensena-japon). 
